\title{
ALKALOIDS FROM Chelidonium majus GROWING IN GEORGIA
}

\author{
N. S. Vachnadze, ${ }^{*}$ A. D. Bozhadze, D. T. Berashvili, \\ A. J. Bakuridze, and V. Yu. Vachnadze
}

UDC 547.945/944

Chelidonium majus L. (Papaveraceae) is broadly distributed over Georgia. It is a perennial herbaceous plant, the alkaloids of which are known for their cytotoxic activity [1]. The alkaloids chelidonine, protopine, L-stylopine, chelerythrine, and berberine were isolated earlier during studies of the species growing in Georgia [2].

Herein we communicate results of further research on the composition of alkaloids in C. majus. The specimen was the aerial part of the plant collected near Tbilisi during seed ripening.

Ground herb $(2 \mathrm{~kg}$ ) was extracted with EtOH by standing at room temperature. The organic solvent was distilled off. The thick extract was treated with $\mathrm{HCl}(5 \%)$ and then made basic using $\mathrm{NH}_{4} \mathrm{OH}(25 \%)$. Alkaloids were extracted with $\mathrm{CHCl}_{3}$ to afford an amorphous total that was separated over a column of silica gel with gradient elution by $\mathrm{CHCl}_{3}: \mathrm{MeOH}(1: 0 \rightarrow 0: 1$, $\mathrm{v} / \mathrm{v}$ ) to afford two bases that were identified based on an analysis of spectral data compared with the literature. ${ }^{1} \mathrm{H}$ and ${ }^{13} \mathrm{C}$ NMR spectra were recorded in $\mathrm{CDCl}_{3}+\mathrm{CD}_{3} \mathrm{OD}$ on a Bruker-400 spectrometer $\left(400 \mathrm{MHz}\right.$ for ${ }^{1} \mathrm{H}$; 100 , for $\left.{ }^{13} \mathrm{C}\right)$.

HPLC analysis of the total was performed on an Agilent-1100 chromatograph using Silicagel-ODS $(250 \times 6 \mathrm{~mm})$, $25^{\circ} \mathrm{C}$, mobile phase $\mathrm{H}_{2} \mathrm{O}+\mathrm{AcCN}(\mathrm{pH} 7.5$ ) at flow rate $1 \mathrm{~mL} / \mathrm{min}$.

Compound 1, white crystalline base, mp 206-208 ${ }^{\circ} \mathrm{C}\left(\mathrm{CHCl}_{3}: \mathrm{EtOAc}\right)$. PMR spectrum $(400 \mathrm{MHz}, \delta, \mathrm{ppm}, \mathrm{J} / \mathrm{Hz})$ : 2.57-2.72 (2H, m, H-6a/H-5a), 2.73-2.89 (1H, t, H-13), 3.02-3.18 (2H, m, H-5b/H-6b), 3.2-3.3 (1H, dd, J = 11.0), 3.47-3.63 $(2 \mathrm{H}, \mathrm{t}), 4.1(1 \mathrm{H}, \mathrm{d}, \mathrm{J}=15.3), 5.7-6.3\left(4 \mathrm{H}, 2 \mathrm{OCH}_{2} \mathrm{O}\right), 6.6(1 \mathrm{H}, \mathrm{s}, \mathrm{H}-4), 6.35(1 \mathrm{H}, \mathrm{d}, \mathrm{J}=8.0, \mathrm{H}-12), 6.69(1 \mathrm{H}, \mathrm{d}, \mathrm{J}=8.0$, H-11), 6.73 (1H, s, H-1). ${ }^{13} \mathrm{C}$ NMR spectrum (100 MHz, $\left.\delta, \mathrm{ppm}\right): 105.42$ (C-1), 144.86 (C-2), 146.07 (C-3), 108.32 (C-4), 127.64 (C-4a), 29.45 (C-5), 51.12 (C-6), 52.80 (C-8), 117.0 (C-8a), 146.07 (C-9).

HPLC analysis of total alkaloids showed that the retention time of chelidonine was 45.559 min; of tetrahydrocoptisine, 47.555; of stylopine, 54.125. A comparison of the results with the literature identified 1 as tetrahydrocoptisine [3-5].

Compound 2, amorphous base. PMR spectrum (400 MHz, $\delta, p p m): 2.5-2.75(2 \mathrm{H}, \mathrm{m}, \mathrm{H}-5 \mathrm{a} / \mathrm{H}-6 \mathrm{~b}), 3.01-3.3$ (2H, m, H-5b/H-6a), 4.8 (6H, s, $\left.2 \times \mathrm{OCH}_{3}\right), 6.0\left(2 \mathrm{H}, \mathrm{s}, \mathrm{OCH}_{2} \mathrm{O}\right), 6.72(1 \mathrm{H}, \mathrm{s}, \mathrm{H}-4), 6.74(1 \mathrm{H}, \mathrm{d}, \mathrm{H}-12), 6.81(1 \mathrm{H}, \mathrm{d}, \mathrm{H}-11), 7.01(1 \mathrm{H}$, s, H-1). ${ }^{13} \mathrm{C}$ NMR spectrum (100 MHz, $\delta$, ppm): 107.24 (C-1), 147.12 (C-2), 147.00 (C-3), 108.72 (C-4), 126.8 (C-4a), 29.43 (C-5), 51.0 (C-6), 54.06 (C-8), 116.5 (C-8a), 149.55 (C-9), 145.67 (C-10), 109.75 (C-11), 123.83 (C-12), 128.0 (C-12a), 36.44 (C-13), 63.0 (C-14), 131.21 (C-14a), $102.31\left(2,3-\mathrm{OCH}_{2} \mathrm{O}\right), 55.78\left(\mathrm{C}-10, \mathrm{OCH}_{3}\right), 59.5\left(\mathrm{C}-9, \mathrm{OCH}_{3}\right)$.

According to an analysis of the results and a comparison with the literature, $\mathbf{2}$ was identified as tetrahydroberberine $[3,5]$.

\section{REFERENCES}

1. J. Lee, M. Y. Slou, D. S. J. Ha, and S. W. Huang, Agric. Chem. Biotechnol., 48, No. 4, 198 (2005).

2. $\quad$ A. Bozhadze, V. Mshvildadze, V. Vachnadze, V. Tsitsishvili, and A. Bakuridze, Khim. Prir. Soedin., 424 (2011).

3. M. Shamma and D. M. Hindenlind, Carbon-13, NMR, Shift Assignments of Amines and Alkaloids, Plenum Press, New York, London, 1979, pp. 141, 144.

4. A. Sazozi, G. Janicsak, L. Kursinski, and A. Kery, Chromatographia, 63, 81 (2006).

5. Kh. N. Aripov (ed.), Progress in Research on Alkaloid-Bearing Plants [in Russian], Fan, Tashkent, 1993, p. 211.

1) Tbilisi State Medical University, Kutateladze Institute of Pharmaceutical Chemistry, Georgia, Tbilisi, 0159, P. Saradzhishvili St., 36, fax: (99532) 5200 23, e-mail: parmhem@yandex.ru; 2) Tbilisi State Medical University, Georgia, Tbilisi, 0162, Prosp. V. Pshavela, 33. Translated from Khimiya Prirodnykh Soedinenii, No. 5, September-October, 2012, p. 815. Original article submitted May 22, 2012. 